\title{
Argentina: el año de Cambiemos ${ }^{*}$
}

\author{
Argentina: The Year of Cambiemos
}

\section{GABRIEL VOMMARO}

CONICET-UNGS, Argentina

\section{MARIANA GENÉ}

CONICET-IDAES/UNSAM, Argentina

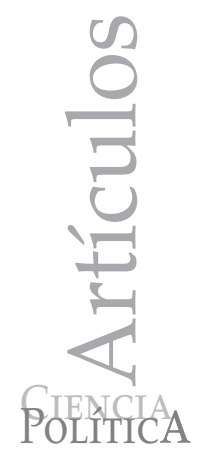

\section{RESUMEN}

Este trabajo analiza el primer año de gobierno de Mauricio Macri, líder de la coalición Cambiemos. Su victoria en 2015 sobre el candidato del entonces gobernante Frente para la Victoria marcó un giro en la política del país. Presentamos los principales acontecimientos políticos, sociales y económicos, así como las líneas fundamentales del nuevo gobierno en esos campos. Mostramos las tensiones de un proyecto de orientación de centro-derecha con tonalidades refundacionales, sustentado en una visión de modernización gerencial de la política y el Estado y de desregulación económica controlada. Este proyecto debe hacer frente a desequilibrios económicos y a pujas sociales estructurales, además de que debe gobernar con minoría parlamentaria y sin control de la mayor parte de los gobiernos provinciales.

Palabras clave: Argentina, giro a la derecha, partidos políticos, políticas públicas, Cambiemos

\begin{abstract}
This paper analyzes the first year of government of Mauricio Macri, leader of the Cambiemos coalition. His victory in 2015 over the candidate of the incumbent Front for Victory marked a turning point in the country's politics. We present the main political, social and economic events of the year and outline the fundamental positions of the new government in relation to these fields. We show the tensions of this center-right reorientation currently unfolding in Argentina, based on a vision of managerial modernization of politics and the state and controlled economic deregulation. We also explain how this project must deal with economic imbalances and social-structural conflicts, in addition to governing with a parliamentary minority and without control of most of the provincial governments.
\end{abstract}

Key words: Argentina, right turn, political parties, public policy, Cambiemos 


\section{INTRODUCCIÓN: LOS DESAFÍOS DEL NUEVO GOBIERNO}

Este trabajo se propone repasar los principales sucesos políticos ocurridos en Argentina durante 2016. En ese tiempo, se cumplió el primer año de gobierno de Mauricio Macri, líder de la coalición Cambiemos, que llegó al poder luego del triunfo en la segunda vuelta de las elecciones presidenciales, el 22 de noviembre de 2015, sobre el candidato del entonces gobernante Frente para la Victoria (FPV), Daniel Scioli ${ }^{1}$. El resultado parecía augurar un profundo giro en la política del país, y esto por diferentes motivos. Por un lado, se trataba del primer triunfo de un candidato que no pertenece ni al Partido Justicialista ni a la Unión Cívica Radical, y que, en cambio, había fundado una fuerza política propia, Propuesta Republicana (PRO), tras la crisis de 2001 y 2002. El quiebre del bipartidismo y la derrota del peronismo luego de doce años de gobierno abría la posibilidad del fortalecimiento de una nueva fuerza política en el país por fuera de las tradicionales, que ya se había consolidado a nivel local a partir de su gestión de gobierno en la ciudad de Buenos Aires (por tres períodos, desde 2007 hasta la actualidad). Por otro lado, la nueva alianza electoral, que logró unificar la mayor parte de las fuerzas políticas no peronistas —dejando afuera solo a la centro-izquierda y a la izquierda de filiación trotskistatiene como socio principal a una fuerza de centro-derecha, que llega al poder por medio de elecciones luego de más de siete décadas de debilidad de las agrupaciones de ese margen del espectro político (Morresi y Vommaro 2014). Por último, las expectativas de cambio político radican en el hecho de que este triunfo electoral se dio luego de un largo ciclo de predominio del peronismo kirchnerista, que, en especial a partir de 2008, se caracterizaba por altas dosis de polarización del debate público y de los discursos partidarios. Basado en una combinación de un discurso nacional-popular reinterpretado a la luz de la tradición del centro-izquierda argentino y de un intenso jacobinismo estatal, el kirchnerismo había dejado progresivamente de lado la agenda republicana - de transparencia y mejoramiento institucional- que había defendido en sus inicios, privilegiando la agenda redistributiva, de ampliación de derechos civiles y culturales y de sostenimiento y regulación estatal del mercado interno, presentándose como "representante del pueblo ante las corporaciones". ${ }^{2}$ Ante este discurso, la oposición recuperó esa agenda republicana "disponible", que pasó a centrarse crecientemente en la denuncia de la corrupción gubernamental y la defensa de una visión liberal-republicana del funcionamiento de las instituciones del Estado. En base a estas banderas, emprendió también, en especial en el caso de PRO y más tarde de sus aliados, una crítica al populismo

\footnotetext{
En la primera vuelta, el 25 de octubre de 2015, Daniel Scioli (FPV) obtuvo el 37,08\% de los votos y Mauricio Macri (Cambiemos) el 34,15\%. En la segunda vuelta, realizada el 22 de noviembre de 2015, Mauricio Macri se impuso con el 51,34\% y Daniel Scioli quedó en segundo lugar con el 48,66\% de los votos.

2 Este giro comenzó a observarse a finales del mandato de Néstor Kirchner, y se aceleró en las presidencias de Cristina Fernández de Kirchner, en especial a partir del conflicto en torno al cambio del régimen de exportación de granos ocurrido en 2008. Sobre el giro dado por el gobierno de Néstor Kirchner, puede consultarse Bonvecchi y Giraudy (2007). Para un análisis del cambio de coyuntura política producido en 2008 y de las respuestas del kirchnerismo a ese cambio, ver Leiras y Cruzalegui (2009).
} 
estatista con que identificó al kirchnerismo, y propuso un "cambio cultural" de carácter refundacional. Como veremos, esta propuesta de cambio, con sus aristas de reorientación económica mercadocéntrica y exportadora, comenzó a ser aplicada en un contexto de restricciones económicas producidas, en buena parte, por la crisis del modelo de crecimiento basado en el consumo interno instituido por el kirchnerismo, y en parte por la decisión del nuevo gobierno de despojarse de recursos económicos con el objetivo de promover la inversión privada. De este modo, paradójicamente, un gobierno que se propone romper con el legado kirchnerista en casi todos los planos debe lidiar con tensiones económico-sociales estructurales que también estaban presentes en el ciclo político anterior $\mathrm{y}$, aunque proponga respuestas muchas veces opuestas en base a una orientación ideológica diferente, no deja de identificar "cuellos de botella" similares. ${ }^{3}$

En las páginas que siguen, analizamos la coyuntura política argentina a la luz de estas cuestiones. En primer lugar, nos ocupamos de los principales acontecimientos políticos, sociales y económicos de 2016, así como de las líneas fundamentales del gobierno de Cambiemos en esos campos, y mostramos el devenir sinuoso de un (nuevo) proyecto con tonalidades refundacionales, sustentado en una visión de modernización gerencial de la política y el Estado y de desregulación económica controlada, que debe hacer frente a desequilibrios económicos y a pujas sociales estructurales. En segundo lugar, nos centramos en la descripción de los vaivenes de la agenda de "cambio cultural" del gobierno, en especial en relación con las continuidades y rupturas en la polarización instalada en el ciclo político anterior, y analizamos la configuración del debate público en torno de las políticas de gobierno en base a la acción del oficialismo, pero también de otros actores relevantes en la materia, como el Poder Judicial. Nos interesa especialmente repasar el modo en que el nuevo gobierno y otros poderes del Estado procesan el ciclo político anterior y proponen un final definitivo para el kirchnerismo. En tercer lugar, nos detenemos en las características del personal político de gobierno: por un lado, en la composición del gabinete ministerial y de las diferentes áreas de gobierno y lo que ella nos dice sobre la empresa de renovación política que busca encarnar Cambiemos, con la incorporación de cuadros del mundo empresario a la gestión del Estado, en consonancia con el proyecto iniciado por PRO (Vommaro et al. 2015); por otro lado, en el estilo de ejercicio del poder presidencial. En cuarto lugar, analizamos la construcción de consensos políticos en tres direcciones: la trabajosa búsqueda de mayorías parlamentarias de un gobierno minoritario en ese terreno, la relación con los gobiernos provinciales, y la consolidación del crecimiento nacional de PRO y de la alianza Cambiemos. Por último, realizamos una evaluación global del primer

El diagnóstico de los cuellos de botella asociados a los problemas cíclicos de las economías latinoamericanas había sido estudiado por la corriente estructuralista en los años 1970. Ver, por ejemplo, el clásico trabajo de Diamand (1973). Mucho se ha dicho sobre la cuestión desde entonces, pero el núcleo del diagnóstico sobre las restricciones de divisas a las que llegaron históricamente los ciclos de redistribución en el continente es casi unánime. 
año de Cambiemos, y de las principales rupturas y continuidades con los rasgos estructurales de la política y la economía argentinas.

\section{2016: TURBULENCIAS ECONÓMICAS Y EXPECTATIVAS POLÍTICAS}

En consonancia con la tradición política argentina de proyectos presidenciales refundacionales (Aboy Carlés 2001), Macri se propuso construir una nueva normalidad en Argentina, en buena parte contrapuesta a la que el kirchnerismo bregó por instaurar desde 2003, y con contornos más definidos a partir de 2008. Y lo hizo en dos sentidos fundamentales: primero, en nombre del destierro de la excepcionalidad y el conflicto como lógica de ejercer el poder; segundo, para producir una modernización económica y social que, en la visión del nuevo presidente y sus aliados, debería acercar al país a su tiempo histórico. La defensa de una concepción política republicana-liberal y de un programa económico-social de modernización de la gestión se convirtieron en las dos banderas de $\mathrm{PRO}$, primero, y de Cambiemos, luego. Como señalamos en otra parte (Vommaro et al. 2015), PRO nació con un proyecto sociocultural y económico que iba en ese sentido, y movilizaba un ethos del voluntariado y el emprenderorismo anclado en el mundo de los negocios y de las ONG, que se proponía llevar al Estado la eficiencia y la transparencia que, en una visión encantada, impera en esos mundos.

Al mismo tiempo, $\mathrm{PRO}$ nace como un partido post-ideológico, con una estrategia flexible y pragmática, que busca construir consensos de gobernabilidad para llevar a cabo su proyecto político. Tiene un diagnóstico claro sobre las dificultades que presentan la sociedad y la economía argentinas para apoyar mayoritariamente esas políticas, es decir, por un lado, los problemas de competitividad de la industria nacional y la tendencia al recalentamiento, en el vocabulario de los años 1970, de las demandas de los sectores populares formales e informales; por otro lado, las estrategias políticas asociadas con esos fenómenos: en ambos casos, por igualitarismo o por corporativismo, actores empresarios y sindicales prefieren los derechos y reaseguros sociales o la negociación mano a mano con el Estado al riesgo emprendedor. Sin embargo, su política de consensos lo llevó, hasta el momento, a preferir el gradualismo antes que las terapias de shock. En líneas generales, el desafío del nuevo gobierno reside en la capacidad de lidiar con esas resistencias y demandas opuestas a su proyecto político, en tiempos de restricciones económicas y sin contar, hasta el momento, con mayoría parlamentaria ni con una abrumadora mayoría electoral.

En esta sección identificamos seis hitos fundamentales de la política argentina en 2016 que permiten comprender las tensiones enfrentadas por el nuevo gobierno, así como el balance entre rupturas y continuidades respecto del ciclo político anterior. 
1. Nueva política de comunicación y nuevo formato de comunicación política. A fines de diciembre de 2015, el nuevo gobierno anunció un decreto — que terminó de hacer oficial a inicios de 2016- que disolvía los dos entes reguladores creados por la Ley de Servicios de Comunicación Audiovisual (en adelante Ley de Medios) y por la Ley de Telecomunicaciones: la AFSCA (Autoridad Federal de Servicios de Comunicación Audiovisual) y la AFTIC (Autoridad Federal de Tecnologías de la Información y la Comunicación), respectivamente. La AFSCA se encargaba de otorgar las licencias de radio y televisión y de regular el mercado de los medios audiovisuales. Era el ente de aplicación de la Ley de Medios, votada por el Congreso en 2010 y producto de un largo debate del que participaron actores del mundo académico y de la sociedad civil. Uno de sus objetivos principales era desconcentrar la propiedad de los medios, y distribuir el espectro audiovisual en partes iguales entre el sector privado, el estatal y el de la sociedad civil. Su implementación parcial, en el contexto de la disputa entre el gobierno de Cristina Fernández de Kirchner y el grupo multimedios Clarín, poco tuvo que ver con el espíritu que le había dado impulso y origen. La supresión por decreto presidencial de la autoridad de aplicación, una de las piezas fundamentales de la ley, estuvo lejos de volver sobre esos pasos. En su lugar, se creó una nueva entidad reguladora, el Ente Nacional de Comunicaciones (ENACOM), que dejó sin representación a las minorías parlamentarias e implicó un mayor control del Poder Ejecutivo sobre el área. La bandera de la democratización fue rápidamente desechada y, en cambio, la preocupación central pasó a ser la modernización tecnológica y el incremento de la competencia de mercado como soluciones a la concentración mediática (Becerra 2016). Esta política fue acompañada de un giro en la comunicación política del gobierno, que pasó a compartir con los grandes grupos multimedios la pedagogía del nuevo programa político y económico, así como la crítica al ciclo kirchnerista. La voz oficial se volvió así más coral, distribuyendo la palabra entre el presidente, algunos de sus ministros y en especial el jefe de gabinete, pero también algunos periodistas y comunicadores. Se trataba, en palabras del jefe de gabinete, Marcos Peña, de "bajarle el volumen" a la voz presidencial después de un tiempo de largos discursos y activa presencia en los medios de la cabeza del Poder Ejecutivo. En esa línea, se evitó la llamada "cadena nacional" — discursos transmitidos por todos los canales de televisión y estaciones de radio- y se apeló a un contacto más emocional con la ciudadanía. En definitiva, la nueva comunicación política quiso ser más difusa y seguir la lógica de propagación de las redes sociales.

2. La deuda como atajo. La política de apertura económica, promoción de las inversiones privadas y reducción de la intervención del Estado en la vida económica eran banderas indiscutidas de la nueva coalición de gobierno. A corto plazo, se trataba de eliminar las restricciones de acceso a las divisas, reducir el déficit fiscal y controlar la inflación. El debate interno tenía que ver con la velocidad de las medidas para avanzar en ese sentido. La eliminación de las regulaciones establecidas por el gobierno de Cristina Kirchner al acceso a las divisas, conocidas como "cepo cambiario", fue realizada rápidamente, a poco de asumir el nuevo gobierno. En cambio, para la reducción de la inflación 
y del déficit fiscal, a comienzos de 2016 se visibilizó un debate dentro de la alianza oficialista entre lo que se llamó, por entonces, gradualistas y partidarios de un shock. Estos últimos promovían medidas inmediatas de disciplinamiento económico y social que suponían una apertura más rápida de las protecciones aduaneras, la reducción de la inversión pública — que incluía reducción del personal estatal en diferentes áreas y recortes en prestaciones sociales- y medidas de disminución de salarios que permitieran reducir la demanda a corto plazo. Los primeros, en tanto, creían que las medidas debían tomarse de modo más paulatino, garantizando, a la vez, consensos políticos —en especial en el ámbito parlamentario - y gobernabilidad social, en relación con posibles protestas sindicales y de movimientos sociales que representan a sectores informales. Aunque había en el plantel del gobierno partidarios de ambas posturas, se impuso el gradualismo para el manejo de las finanzas y la producción —el Ministerio de Hacienda- y se concedió el manejo de la política monetaria - la presidencia del Banco Central- a los más ortodoxos. Las tensiones entre los dos comandos de la economía se mantendrían a lo largo de todo el año. Para los gradualistas, era necesario obtener recursos para financiar un gasto público que se mantendría elevado sin recurrir a la emisión monetaria.

El acceso al crédito internacional estaba parcialmente dificultado por el conflicto que mantenía el Estado argentino con los llamados "fondos buitre". El gobierno de Cambiemos inició una negociación a comienzos de año con esos fondos, que culminó con la aprobación de una ley en el Congreso que derogaba leyes anteriores que bloqueaban un acuerdo y con la autorización para tomar deuda en el exterior por 12.500 millones de dólares. Así, en pocos meses, logró una victoria política, mostró su capacidad de construir consensos parlamentarios y consiguió fondos para financiar gastos corrientes durante ese año. Como puede verse en el Cuadro 1, el aumento del ritmo de endeudamiento durante 2016, que acelera una tendencia que ya había comenzado en 2014 (Murillo et al. 2016), da cuenta de este atajo que permite evitar el aumento de la conflictividad social al tiempo que crea nuevos condicionamientos para la política económica futura. Asimismo, en 2016 el gobierno echó mano a otro recurso habitual de los gobiernos argentinos para acceder a recursos extraordinarios: organizó un proceso de exteriorización de patrimonio - llamado "blanqueo" - que o bien estaba por fuera del sistema bancario o bien registrado en el exterior, y por tanto fuera de la órbita de la agencia recaudadora argentina. El ingreso de divisas a través del blanqueo permitió también mejorar el stock de dólares en el mercado financiero local, factor clave para mantener su precio bajo y contener la inflación.

La presión sobre el descenso de la inversión pública en áreas sociales se volverá, probablemente, un tema de primer orden en la agenda social, más aún cuando la caída de la actividad económica - y la baja de impuestos a actividades primarias, como el agro y la minería- continúe impactando negativamente sobre la recaudación de impuestos. Asimismo, en tanto la estrategia de poner la suerte de la reactivación económica en manos de la inversión privada se mostró 
errada, al menos a corto plazo, ${ }^{4}$ la inversión del Estado en infraestructura se volvió — del mismo modo que lo era en los últimos años del ciclo kirchneristael motor principal de la actividad económica.

Cuadro 1. Principales indicadores económicos y sociales, 2016

\begin{tabular}{lrrr}
\hline & \multicolumn{1}{c}{2014} & \multicolumn{2}{c}{2015} \\
\hline PBI (\% var. anual) & $-2,5$ & 2,4 & $-2,3$ \\
Consumo real (\% var. anual) & $-1,1$ & $-0,7$ & $-3,3$ \\
Deuda externa (\% del PBI) & $42,8 \%$ & $43,7 \%$ & $50 \%$ \\
Inflación (promedio IPC CABA y San Luis) & $40,8 \%$ & $29,3 \%$ & $36,2 \%$ \\
Tipo de cambio & 8,6 & 13,0 & 15,7 \\
Desempleo (\% de la PEA) & $7,3 \%$ & $6,5 \%$ & $8,5 \%$ \\
Pobreza & $28,7 \%$ & $29,0 \%$ & $32,9 \%$ \\
\hline
\end{tabular}

Fuente: Elaboración propia en base a datos del BCRA, INDEC, Dirección Provincial de Estadísticas y Censos, San Luis, Dirección General de Estadísticas y Censos, CABA, Observatorio de la Deuda Social Argentina, UCA, CEPAL.

Nota: Se privilegió mantener series de datos comparables. Por eso, aun cuando a partir de 2016 existen cifras del INDEC en distintas materias, cuando no están disponibles para los años anteriores (i.e., pobreza, inflación) se utilizaron otras fuentes.

3. La vuelta al mundo. Hasta 2015, los socios de Cambiemos no habían podido dar cuenta de una orientación precisa en materia de política exterior, por gobernar distritos subnacionales. Las relaciones internacionales del partido dominante de la coalición, PRO, lo acercaban a las posiciones de la internacional de centroderecha de la que forma parte, y en especial con el Partido Popular español (Vommaro et al. 2015). Sin embargo, en tanto estas alianzas no habían tenido hasta entonces consecuencias de política concretas, la llegada de Cambiemos al gobierno nacional abría un interrogante sobre los alcances de la ruptura con la política exterior del kirchnerismo. Y esto en dos sentidos: respecto de la relación política con los gobiernos de la región y de la política comercial con China, Rusia y otros mercados alternativos. La elección de una canciller moderada auguraba cierto gradualismo también en este campo. En términos de política regional, el gobierno de Macri, que fue parte de la ola de "giro a la derecha" en Sudamérica, encabezó las críticas al gobierno de Nicolás Maduro en Venezuela y a la cada vez más deteriorada situación política de ese país. Al mismo tiempo, el posicionamiento de Macri como defensor de los derechos humanos en ese caso se vio en cierta medida contrarrestado por la situación de la dirigente social

4 La inversión total cayó en un 4\% en 2016, según el INDEC, y acompañó la caída del PIB. Esta caída se manifestó tanto en la inversión pública como en la privada. Todos los analistas económicos coincidieron que, en virtud de estos resultados, "la puesta en marcha de las inversiones anunciadas ha sido más lenta que lo previsto", como afirma un informe de coyuntura del Área de Research del banco BBVA preparado en el primer trimestre de 2017. Lo previsto refería al pensamiento gubernamental, pero también de muchos analistas económicos, respecto de que la llegada de un gobierno promercado aceleraría inversiones privadas demoradas con las incertidumbres generadas al final del ciclo kirchnerista, hecho que no tuvo lugar. 
kirchnerista Milagro Sala, presa en la provincia de Jujuy, donde era la principal referente del movimiento social Tupac Amaru, en condiciones criticadas por el amplio arco de organismos de derechos humanos en Argentina y por organismos internacionales como la Comisión Interamericana de Derechos Humanos. ${ }^{5}$ Este representó un problema para la diplomacia del país que aún no se ha resuelto. En cuanto a la política comercial, la orientación inicial de privilegio de acuerdos con países de mayor apertura de sus fronteras aduaneras, expresada en el ingreso de Argentina como miembro observador a la Alianza del Pacífico puerta de entrada a acuerdos comerciales con Estados Unidos-, así como en el avance hacia la firma de tratados de libre comercio con la Unión Europea, parecía dar cuenta de un giro más radical de la política exterior argentina. Sin embargo, los resultados de las elecciones presidenciales norteamericanas y el viraje proteccionista de ese país, así como las dificultades para avanzar en acuerdos de libre comercio con la Unión Europea, llevaron al gobierno de Macri a privilegiar la ya instalada alianza estratégica e histórica con Brasil, y la no menos estratégica pero más reciente con China. La política exterior oscila así entre un nuevo posicionamiento ideológico y un pragmatismo continuista. ${ }^{6}$

4. Reducción de subsidios a servicios públicos y tensiones con las clases medias. Otra área en la que la política de reducción del gasto público se encontró rápidamente con resistencias organizadas y no organizadas de la sociedad argentina fue la de los subsidios estatales a las tarifas de servicios públicos. La política de tarifas del kirchnerismo había significado en los hechos un subsidio indiscriminado —es decir, sin direccionalidad ni criterio de equidad — tanto para el presupuesto de los hogares como para los diferentes sectores de la economía. El costo a pagar, cada vez más visible y oneroso, fue la caída de la inversión en el sector energético y el deterioro de buena parte de esos servicios. El gobierno de Cambiemos quería, a la vez, reducir el gasto del Estado en ese rubro y asegurar un aumento de la rentabilidad a las empresas proveedoras de servicios para promover la inversión privada. Utilizó un frame que se extendería luego a otras áreas de la economía: el del "sinceramiento", que suponía que, frente al "engaño populista"7 anterior, era

\footnotetext{
Milagro Sala es la principal referente del movimiento Tupac Amaru, con epicentro en Jujuy. Este se había fortalecido durante el ciclo kirchnerista en virtud del despliegue de políticas de bienestar paraestatales, con apoyo financiero del Estado nacional, que había desarrollado políticas de sostenimiento y promoción de formas de organización cooperativas de los movimientos de pobres informales en diferentes lugares del país. Sala fue denunciada por el nuevo gobernador de Cambiemos en la provincia por haber llevado a cabo una manifestación frente a la oficina del Poder Ejecutivo de Jujuy. Fue detenida el 16 de enero de 2016 y, a pesar de haberse dictado su excarcelación por esta causa, a continuación se iniciaron otras acciones penales en su contra, vinculadas con malos manejos de los fondos públicos recibidos, pero también con amenazas y violencia sobre sus adversarios, en virtud de las cuales la justicia provincial decidió mantener la prisión preventiva. La Comisión Interamericana de Derechos Humanos (CIDH) y el Grupo de Trabajo de Naciones Unidas sobre Detención Arbitraria sostuvieron que la detención fue arbitraria y violatoria de los estándares del Pacto Internacional de Derechos Civiles y Políticos, del cual Argentina es parte. Ver CIDH (2016).

6 Como sostiene Andrés Malamud, "el mundo que Macri enfrenta es el mundo que Cristina preveía", es decir un norte proteccionista y mercados abiertos en China y Rusia. Ver Rosso (2016, 8 de diciembre).

El engaño populista. Por qué se arruinan nuestros países y cómo rescatarlos (Planeta, 2016) es precisamente el título de un libro publicado el año pasado que tuvo gran repercusión en ámbitos intelectuales identificados con el centro-derecha latinoamericano. La guatemalteca Gloria Álvarez, autora junto al chileno Axel Kaiser, estuvo en Argentina en 2015 y apoyó la candidatura de Macri.
} 
necesario pagar los costos que suponía "mirar la realidad de frente" y pensar en el largo plazo. Aunque la mayor parte de la población aceptaba un ajuste en las tarifas de los servicios, la magnitud del aumento - de hasta 500\% - encontró rápidamente oposición en el activismo de clases medias, que utilizó los recursos con los que enfrenta habitualmente las acciones del Estado: amparos judiciales y manifestaciones callejeras inorgánicas tipo "cacerolazos". Se trató de una de las primeras derrotas políticas del gobierno, que debió reorganizar el cronograma de aumentos y aplicar, aquí también, una política gradualista. Asimismo, el llamado "tarifazo" puso en cuestión la sensibilidad política de los cuadros de gobierno provenientes del mundo empresario.

5. Emergencia ocupacional. La apertura de las importaciones en algunos rubros, así como la caída del consumo, produjeron desde el primer trimestre de 2016 pérdida de empleos, en especial en el sector industrial y de la construcción. Esta situación se revirtió parcialmente en la segunda mitad del año. Sin embargo, la recuperación se basó, según el Ministerio de Trabajo, en el aumento del empleo público y de los trabajadores independientes registrados (monotributistas), que compensaron parcialmente la caída del empleo privado formal, calculada en poco más de 43 mil puestos de trabajo. Según el Instituto Nacional de Estadísticas y Censos (INDEC 2017), entre el tercer trimestre de 2015 y el tercer trimestre de 2016 se redujo el empleo registrado en un 1,5\% y la cantidad de empresas registradas en un $0,7 \% .{ }^{9}$ A estas cifras debe sumarse, según los especialistas, la pérdida de al menos la misma cantidad de puestos de trabajo no registrados, en la llamada economía informal. Este deterioro del mercado del trabajo se dio en el contexto de una caída del poder adquisitivo del salario de entre un 6 y un $8 \%$, a causa de la inflación. Ante esta situación, el sindicalismo, de raíz mayoritariamente peronista, ensayó diferentes estrategias de presión, que fueron desde la propuesta de una Ley de Emergencia Laboral — por la cual se suspendían los despidos por 180 días, y se establecía la doble indemnización en caso de que se concretaran-, votada por el Parlamento pero luego vetada por el Poder Ejecutivo, hasta la organización de una movilización masiva el 29 de abril de 2016 en apoyo a esa medida. A pesar de tomar caminos no intervencionistas en términos de regulación del empleo, y por tanto opuestos a la matriz ideológica arraigada en la mayor parte de las vertientes del movimiento sindical argentino, el gobierno logró controlar las tensiones con ese movimiento. Así, consiguió terminar el año sin paros nacionales - lo que no había ocurrido con los anteriores gobiernos no peronistas del actual ciclo democrático-, pero a cambio tuvo que distribuir recursos entre los sindicatos, en especial para financiar sus prestaciones de salud - como había hecho el reformismo menemista en los años noventa (Etchemendy 2001). En líneas generales, la tensión con un sindicalismo organizado y fuerte no es novedad en la Argentina reciente. El kirchnerismo, que

Ver Fernández Blanco (2017).

Cabe destacar que el INDEC recuperó parte de su capacidad técnica y su autonomía relativa en el segundo semestre de 2016, en base a una decisión gubernamental de recobrar la confiabilidad de los números públicos. 
contribuyó a removilizar políticamente un sindicalismo que había sobrevivido durante los años noventa en base a mantener su poder organizativo (Murillo 1997), terminó enfrentado a algunos de sus sectores, en especial por la carga impositiva de los salarios. Las tensiones que se avizoran en el escenario actual tienen que ver no solo con las demandas sectoriales sino también con los efectos de las políticas económicas en los sectores asalariados. En este sentido, el actual gobierno considera que el llamado "costo laboral" es un freno al crecimiento de la inversión privada en Argentina. Comparte este diagnóstico con buena parte del mundo empresario. ${ }^{10}$ Sin embargo, en su primer año el gobierno avanzó muy poco en su proyecto de reducción del costo de la mano de obra, que es probablemente una de las grandes directrices para el resto de su gestión. En qué medida ese lineamiento chocará con la oposición del sindicalismo será materia de futuros análisis.

6. Emergencia social. El otro problema que debió enfrentar el nuevo gobierno en relación con las clases populares es la política hacia los sectores informales, nudo gordiano de la nueva cuestión social en Argentina (Vommaro 2017). El kirchnerismo había consolidado una forma de bienestar precario para ese porcentaje de la población económicamente activa, que oscila entre un cuarto y un tercio de ella, según estimaciones, y que constituye la masa de trabajadores sin empleo estable, empleada en "changas", cuyo presupuesto proviene en buena parte de los diferentes programas sociales implementados por el kirchnerismo a partir de 2009: el programa de financiamiento de cooperativas conocido como Argentina Trabaja y la Asignación Universal por Hijo (AUH), de carácter cuasi-universal. El gobierno de Cambiemos heredó ese estado del mercado de trabajo, con una fuerte proporción de informales, así como las políticas destinadas a proveer bienestar a ese sector. Mantuvo las dos líneas de financiamiento del mundo popular durante 2016, y hasta amplió los grupos beneficiarios de la AUH (Niedzwiecki y Pribble 2017). Dio cuenta así de que está dispuesto a mantener el gasto público en esta área. Al mismo tiempo, los movimientos sociales que organizan a los sectores informales, y que se han vuelto co-gestores de políticas sociales a nivel local, ejercieron presión sobre el nuevo gobierno para, por un lado, continuar avanzando en un reconocimiento institucional del sector que esos grupos llaman de "economía popular" y, por otro lado, garantizar la continuidad del financiamiento estatal hacia él a través de los programas de apoyo a cooperativas. La movilización y los piquetes en apoyo a una Ley de Emergencia Social convencieron al gobierno de la necesidad de mantener estas políticas ante la amenaza de un aumento de la conflictividad

10 Por ejemplo, ante la pregunta sobre la realización de nuevas inversiones, Carlos Gomes, presidente de la automotriz PSA para América Latina, respondió: "En el plano económico se necesita confianza y la confianza se gana poco a poco. Venimos de un período difícil donde hemos sufrido bastante. Hoy necesitamos que esto se establezca y se oriente. Las condiciones [para invertir] hoy todavía no son excelentes". Entre esas condiciones se encuentra, consignó el CEO, "el costo de la mano de obra", que es "más alto que en Brasil". Entrevista a Carlos Gomes: "Carlos Gomes: 'No creo que la economía argentina pueda desplegarse todavía"”, 4 de mayo de 2016. Recuperada el 1 de agosto de 2017 de: http:/ / www.lanacion.com.ar/1895214-carlos-gomes-no-creo-que-la-economia-argentina-pueda-desplegarse-todavia 
social. Muchas y diversas organizaciones sociales visitan asiduamente la Casa de Gobierno y tienen canales abiertos para expresar sus demandas. El gobierno tiene como desafío, no obstante, mantener esta interlocución con un actor que posee pertenencias políticas más bien opuestas a las orientaciones de Cambiemos, en un contexto en que se profundizará la política de reducción del déficit fiscal y no parece haber señales de reactivación del mercado del trabajo. En definitiva, el gobierno de Cambiemos atravesó 2016 con una combinación entre intentos de reforma, manejo de problemas heredados y producidos por la nueva política, y mantenimiento de la gobernabilidad política y social. La paradoja, según muestran la mayor parte de los estudios de opinión, es que la administración Macri logró concitar grandes expectativas hacia el futuro aun cuando la mayor parte de la población consideraba negativa la situación económica. ${ }^{11}$ En 2016, así, logró armonizar la percepción de mal desempeño económico con alta expectativa política. La apuesta a un futuro promisorio a costa de esfuerzos en el presente se reveló exitosa en el contexto de una fuerte crisis de la imagen pública del kirchnerismo y de la división del peronismo en diferentes facciones. La crítica a un populismo pasado que había garantizado beneficios inmediatos a costa de problemas futuros contrastó con la austeridad "sincera" que propuso el nuevo gobierno. Sin embargo, fue necesario conceder protecciones sociales y aumentos salariales que compensaran los problemas económicos. En definitiva, posponer el programa de austeridad que debía sentar las bases de un crecimiento económico futuro. Esta tensión se agudizará durante 2017, año electoral, y será uno de los temas de la segunda mitad del mandato de Cambiemos.

\section{EL PODER JUDICIAL COMO ACTOR POLÍTICO Y LAS CAUSAS DE CORRUPCIÓN}

Durante 2016 distintos miembros del Poder Judicial fueron actores centrales de la escena política en Argentina. Ese protagonismo se inscribe en un tiempo largo en el que, por un lado, se afianzaron procesos de "judicialización de la política" en distintas regiones del mundo (Smulovitz 2008), y por el otro, los conflictos abiertos entre el gobierno de Cristina Kirchner y el sistema judicial vieron cambiar su escenario institucional con el traspaso de mando.

El gobierno de Macri realizó distintos movimientos para relacionarse con este poder. Por un lado, a pocos días de asumir, el presidente designó por decreto a dos jueces de la Corte Suprema de Justicia en comisión, hasta tanto fueran aprobados por el Senado. Esa iniciativa chocó con críticas de distintos sectores políticos y de la sociedad civil, por lo que su trámite debió volver a los carriles

11 Durante todo 2016, los que evaluaban como positiva la situación económica del país oscilaron entre el 12\% y el $20 \%$, mientras que las expectativas de mejora económica para el siguiente año fueron de entre el $43 \%$ y el 57\% (y para los siguientes tres años el optimismo aumentó a valores de entre el $48 \%$ y el $64 \%$ ). En cuanto a la situación general del país, quienes lo veían bien oscilaron entre el 15 y el 24\%, pero al indagar por sus expectativas a futuro, durante el año el optimismo alcanzó entre el 52 y el 69\% (Poliarquía 2016). 
normales y ambos candidatos fueron oficializados en sus cargos en junio de 2016. También en el Consejo de la Magistratura, órgano encargado de la administración general del Poder Judicial y de la selección y acusación de los jueces, el gobierno tuvo una estrategia contundente. Aquel órgano, que había tenido mayoría kirchnerista hasta fines de $2015,{ }^{12}$ viró en pocos meses hacia una mayoría alineada con el gobierno de Macri. Por medio de una interpretación de su normativa interna que aún espera ser confirmada y de las elecciones en cuyo armado de listas participó activamente el ministro de Justicia, el oficialismo alcanzó la minoría simple de ese órgano en septiembre. Desde entonces, el presidente instó en distintas ocasiones a sus miembros a que motoricen los juicios políticos abiertos para destituir a algunos jueces. Dicho proceso todavía está en curso y su interpretación es polémica: desde el gobierno argumentan que la estrategia busca responder al "reclamo social" de mayor transparencia en todos los poderes del Estado, pero sus detractores señalan que se promueven las causas contra los jueces no alineados con el gobierno. Similares debates tuvieron lugar entre los partidarios de destituir a la Procuradora General de la Nación y quienes apoyaron su continuidad. La jefa de los fiscales fue nombrada durante el kirchnerismo en un cargo que al igual que los de la Corte Suprema es por tiempo indeterminado, y desde el nuevo gobierno se intentó primero obtener su renuncia mediante negociaciones encabezadas por el ministro de Justicia, luego sancionar una ley que estableciera la duración de su mandato en 5 años, que se encontró con el escollo de que su aplicación no podía ser retroactiva, y por último promover pedidos de juicio político para destituirla.

Lo cierto es que el tema de la relación entre el poder político y el Poder Judicial ocupó un lugar importante en la agenda nacional durante todo el año. Esto se vio ampliamente potenciado por los juicios contra la expresidenta por causas de corrupción, que jalonaron la agenda política. Cristina Kirchner fue citada a declarar en los tribunales de Comodoro Py cuatro veces en el año por distintas causas. Tales acontecimientos fueron espacios de encuentro de la militancia kirchnerista y de reafirmación de su identidad política, con movilizaciones masivas - especialmente la primera-y discursos de la expresidenta fustigando las políticas llevadas adelante por el gobierno de Macri y denunciando una persecución político-judicial en su contra. A la vez, fueron una oportunidad para la reafirmación de la identidad anti-kirchnerista y para la diferenciación del gobierno y sus aliados respecto del pasado reciente. Si bien el presidente se refirió muchas veces a la necesidad de "cerrar la grieta" que dividía a los argentinos, también aprovechó esa polarización para afianzarse y resguardar su popularidad en momentos en que los resultados macroeconómicos y sociales no lo garantizaban, y la identificación del kirchnerismo como un todo con la corrupción fue una de las principales estrategias en ese sentido. Precisamente,

12 Sus trece miembros se reparten de forma colegiada: dos abogados, tres jueces, tres diputados y tres senadores (dos del oficialismo y uno de la oposición en ambos casos), un miembro de las universidades y un representante del Ejecutivo. En 2013 el gobierno de Cristina Kirchner intentó una reforma del Consejo que ampliaba la cantidad de miembros y hacía que la mayoría de ellos se eligieran por medio de sufragio universal, pero la Corte declaró su inconstitucionalidad. 
la corrupción fue un tema central del debate político y distinto tipo de actores -fundamentalmente políticos, expertos y periodísticos- encontraron públicos ya entrenados en (y habituados a) el consumo de información sobre ilegalismos cometidos por funcionarios (Pereyra 2013).

Los jueces federales fueron decisivos en este proceso, y seguramente también lo fue el largo conflicto entre los gobiernos de Cristina Kirchner y el Poder Judicial, que había tenido lugar al menos desde 2010. Entre sus episodios más salientes se encontraron el intento de reforma del Consejo de la Magistratura, las tensiones con la Corte Suprema por los plazos para expedirse sobre la constitucionalidad de la Ley de Medios, y la denuncia del surgimiento de un "partido judicial" que actuaba por fuera de las fronteras constitucionales obstruyendo decisiones políticas. Con el cambio de gobierno, algunos de los letrados mostraron sus habilidades para manejar el timing de las causas, produciendo acontecimientos con grandes repercusiones en momentos políticamente significativos. Los múltiples contactos de magistrados con políticos - o con sus operadores- y con los servicios de inteligencia renovaron una vez más las dudas que pesan sobre la independencia judicial, en especial en el decisivo fuero federal (Hauser 2016). En cualquier caso, el judicial fue un poder de peso en la política y lo seguirá siendo durante 2017, en tiempos de elecciones legislativas en que la situación procesal de la expresidenta será uno de los elementos que definirá ese tablero.

\section{EL NUEVO GABINETE Y EL ESTILO PRESIDENCIAL}

La llegada al gobierno de Cambiemos produjo también una modificación en los elencos ministeriales, que representa una marca de distinción para los oficialistas y un foco de críticas para los opositores. En efecto, el perfil de los elencos de gobierno de Cambiemos es sensiblemente diferente al de los gabinetes anteriores del actual ciclo democrático. Nuevos cuadros políticos llegaron al Ejecutivo nacional, en consonancia con los mundos sociales en que PRO había reclutado su personal político, tanto a través de mediaciones institucionales formales (ONG y Fundaciones) como informales (relaciones personales) construidas por esa fuerza política antes de la llegada al poder (Vommaro et al. 2015).

El gabinete de Macri tiene cuatro tipos fundamentales de personal político: los cuadros formados en el gobierno de la Ciudad de Buenos Aires y en sus instituciones asociadas, como la Fundación Pensar y el Banco Ciudad —donde se desarrollaron buena parte de los economistas que administran los recursos en los tres gobiernos de PRO: el nacional, el de la provincia de Buenos Aires y, desde luego, el de la Ciudad Autónoma-; los CEO de grandes corporaciones conectadas con diferentes mercados internacionales; los expertos de áreas específicas; y los cuadros políticos de partidos aliados (ver Cuadro 2). En términos políticos, sin embargo, esta complejidad puede reducirse a dos dimensiones. Por un lado, prevalece el control partidario por sobre la lógica de 
coalición, ya que la mayor parte de los ministros y secretarios provienen de PRO y de sus fundaciones. A la hora de conformar el gabinete el presidente hizo valer las prerrogativas que el sistema presidencialista le acuerda, y que el equilibrio de poder en el interior de la coalición le permitió ampliamente. De hecho, frente al fantasma de la Alianza (entre la UCR y el Frepaso) que vio su fin en la crisis de 2001, Macri afirmaría tempranamente que el suyo no sería un gobierno de coalición: "el que gana va a gobernar". ${ }^{13}$ En este sentido, hay tres ministros de la UCR en un gabinete que cuenta con 23 carteras, pero además esos radicales no fueron elegidos por el partido centenario según sus reglas internas sino por las preferencias del presidente: ${ }^{14}$

\section{Cuadro 2. Gabinete nacional, 2016}

\begin{tabular}{|c|c|c|c|}
\hline Ministerio* & Ministro & Sector & Cargo anterior \\
\hline Jefatura de Gabinete & Marcos Peña & PRO & $\begin{array}{l}\text { Secretario General de la } \\
\text { Ciudad de Bs. As. }\end{array}$ \\
\hline Interior & Rogelio Frigerio & PRO & $\begin{array}{l}\text { Presidente del Banco Ciudad } \\
\text { de Bs. As. }\end{array}$ \\
\hline Rel. Exteriores y Culto & Susana Malcorra & Expertos & $\begin{array}{l}\text { Jefa de Gabinete de la } \\
\text { Secretaría general de las } \\
\text { Naciones Unidas }\end{array}$ \\
\hline Defensa & Julio Martínez & $\begin{array}{l}\text { Aliados PRO } \\
\text { (UCR) }\end{array}$ & Diputado nacional \\
\hline $\begin{array}{l}\text { Hacienda y Finanzas } \\
\text { Públicas } \\
\text { (hasta 26/12/2016) }\end{array}$ & $\begin{array}{l}\text { Alfonso Prat } \\
\text { Gay }\end{array}$ & Aliados PRO & $\begin{array}{l}\text { Consultor } \\
\text { Antes diputado nacional }\end{array}$ \\
\hline $\begin{array}{l}\text { Hacienda } \\
\text { (desde 27/12/2016) }\end{array}$ & Nicolás Dujovne & Expertos & Consultor \\
\hline $\begin{array}{l}\text { Finanzas } \\
\text { (desde 27/12/2016) }\end{array}$ & Luis Caputo & Expertos & Secretario de Finanzas \\
\hline Producción & $\begin{array}{l}\text { Francisco } \\
\text { Cabrera }\end{array}$ & PRO & $\begin{array}{l}\text { Ministro de Desarrollo } \\
\text { Económico de la Ciudad de } \\
\text { Bs. As. }\end{array}$ \\
\hline Turismo & Gustavo Santos & $\begin{array}{l}\text { Aliados PRO } \\
\text { (Schiaretti, PJ) }\end{array}$ & $\begin{array}{l}\text { Presidente de la Agencia } \\
\text { Córdoba Turismo }\end{array}$ \\
\hline Modernización & Andrés Ibarra & PRO & $\begin{array}{l}\text { Ministro de Modernización de } \\
\text { la Ciudad de Bs. As. }\end{array}$ \\
\hline
\end{tabular}

13 Ver Diario Popular (2015): “Macri no quiere un gobierno de coalición con la UCR", 17 de marzo de 2015.

14 La lógica de coalición se aplica, en cambio, a nivel parlamentario —el radicalismo provee más del $40 \%$ de los diputados del interbloque de Cambiemos- y a nivel subnacional, a través de la aplicación de políticas en provincias y municipios. Cf. al respecto Cruz y Goyburu (2016). 


\begin{tabular}{|c|c|c|c|}
\hline Ministerio* & Ministro & Sector & Cargo anterior \\
\hline Seguridad & Patricia Bullrich & $\begin{array}{l}\text { Aliados PRO } \\
\text { (Unión por la } \\
\text { Libertad) }\end{array}$ & Diputada nacional \\
\hline Justicia y DDHH & $\begin{array}{l}\text { Germán } \\
\text { Garavano }\end{array}$ & Expertos & $\begin{array}{l}\text { Consejero suplente del } \\
\text { Consejo de la Magistratura }\end{array}$ \\
\hline Trabajo & Jorge Triaca & PRO & Diputado nacional \\
\hline Salud & Jorge Lemus & PRO & $\begin{array}{l}\text { Ministro de Salud de la } \\
\text { Ciudad de Bs. As. (hasta 2012) }\end{array}$ \\
\hline Desarrollo Social & Carolina Stanley & PRO & $\begin{array}{l}\text { Ministra de Desarrollo Social } \\
\text { de la Ciudad de Bs. As. }\end{array}$ \\
\hline Educación & Esteban Bullrich & PRO & $\begin{array}{l}\text { Ministro de Educación de la } \\
\text { Ciudad de Bs. As. }\end{array}$ \\
\hline Ciencia y Tecnología & Lino Barañao & Expertos & $\begin{array}{l}\text { Ministro de Ciencia y } \\
\text { Tecnología }\end{array}$ \\
\hline Cultura & Pablo Avelluto & PRO & $\begin{array}{l}\text { Coordinador General de } \\
\text { Medios Públicos de la Ciudad } \\
\text { de Bs. As. }\end{array}$ \\
\hline Agroindustria & Ricardo Buryaile & $\begin{array}{l}\text { Aliados PRO } \\
\text { (UCR) }\end{array}$ & Diputado nacional \\
\hline Transporte & $\begin{array}{l}\text { Guillermo } \\
\text { Dietrich }\end{array}$ & PRO & $\begin{array}{l}\text { Subsecretario de Transporte } \\
\text { de la Ciudad de Bs. As. }\end{array}$ \\
\hline Energía & $\begin{array}{l}\text { Juan José } \\
\text { Aranguren }\end{array}$ & Expertos & Presidente de Shell Argentina \\
\hline Medio Ambiente & Sergio Bergman & $\mathrm{PRO}$ & Diputado nacional \\
\hline Comunicaciones & Oscar Aguad & $\begin{array}{l}\text { Aliados PRO } \\
\text { (UCR) }\end{array}$ & Diputado nacional \\
\hline
\end{tabular}

* Los nombres de algunas carteras están abreviados.

Fuente: elaboración propia en base a web de Presidencia de la Nación y diferentes medios de prensa.

Por otro lado, la inclusión de CEO y managers en las primeras y segundas líneas del gabinete refuerza la importancia que ha tenido para PRO el mundo de la empresa como espacio de reclutamiento de personas y repertorios de gestión y de acción (Vommaro et al. 2015). Además de los miembros del partido que provienen del mundo empresario (Cabrera, Dietrich), están los CEO que debutan en el gabinete, como Juan José Aranguren (ex CEO de Shell, en Energía y Minería), Gustavo Lopetegui y Mario Quintana (ex CEO de LAN y de Farmacity respectivamente, coordinadores de la Jefatura de Gabinete). Más allá de su novedad, atractiva para el análisis mediático, los managers son puestos en primer plano en la presentación del nuevo gabinete por el propio personal político de PRO, que tomó cada ingreso de un cuadro empresario al gobierno como el logro de un "pase" en un mercado hipercompetitivo. Tal como en la identidad partidaria se invisibiliza a los cuadros políticos de larga data en pos 
de su construcción como un partido nuevo de quienes se "meten en política" (Vommaro y Armesto 2015), el gobierno de Cambiemos elige ahora poner de relieve esa dimensión del "cambio cultural" que se expresa a través de sus managers.

La centralidad de la Jefatura de Gabinete es otra de las innovaciones del primer año de gobierno de Macri. Está encabezada por uno de sus hombres de máxima confianza y miembro histórico del PRO, Marcos Peña, quien condujo la campaña presidencial en términos de comunicación ${ }^{15}$ y ocupa actualmente un rol fundamental de spin doctor, a la vez intérprete y estratega del gobierno, responsable de marcar el "tono" de la gestión. Se encuentra además secundado por los dos CEO nombrados más arriba -Quintana y Lopetegui-, que se encargan del seguimiento del presupuesto asignado a cada uno de los ministerios. Su relación con lo que desde el Poder Ejecutivo denominan los "equipos" es constante y a veces tensa, como pusieron en evidencia los recambios importantes ocurridos durante 2016, como el de Alfonso Prat Gay de la cartera de Hacienda y Finanzas, o el de Isela Constantini (ex CEO de General Motors) de Aerolíneas Argentinas.

La salida de Prat Gay del principal ministerio económico ${ }^{16}$ se consumó durante los últimos días de diciembre y fue interpretada masivamente como un fortalecimiento de los tres miembros que encabezan la Jefatura de Gabinete. Prat Gay había llegado al gobierno como un exponente del gradualismo frente a quienes promovían políticas de shock, pero tras un conflicto en torno a la modificación del impuesto a las ganancias fue removido y su cartera dividida en dos. Desde entonces entraron en funciones un ministro de Hacienda -Nicolás Dujovne, consultor económico y promotor más férreo de la reducción del déficit y los subsidios - y otro de Finanzas - Luis Caputo, proveniente del mundo de las finanzas y los bancos, y de cercanía personal con el presidente- más alineados con el programa presidencial.

Finalmente, el único ministro que se mantuvo del ciclo kirchnerista fue el de Ciencia y Técnica. Aquel Ministerio, creado al iniciarse el primer gobierno de Cristina Kirchner, tuvo a Lino Barañao a la cabeza durante ocho años, y su mantenimiento con el traspaso de mando fue una novedad y una sorpresa. Los recientes recortes en esa área producidos hacia fines de 2016 hacen entrever que la continuidad de personas no indica necesariamente continuidad de las políticas.

También la figura presidencial vivió transformaciones en el primer año de gobierno de Cambiemos. El formato de la comunicación política y la estética de presentación pública que PRO desarrolló en sus años previos a la llegada

15 Por su parte, Emilio Monzó fue el encargado de tejer las principales alianzas políticas en todo el territorio. Para una crónica "desde adentro" de esa campaña, ver Iglesias Illa (2015).

16 La cartera de Hacienda y Finanzas convivió con otros ministerios que antes formaban parte de Economía como los de Producción, Energía o Agroindustria. 
al gobierno nacional continuaron en cierta medida en esta nueva etapa. Se observó así un modo informal de comunicación y la movilización de índices de cercanía con los ciudadanos - referirse a personas comunes en los discursos y llamarlas por su nombre de pila, realizar "timbreos" y visitas personalizadas-, así como una impronta optimista que sustenta un discurso que subraya ideas asociadas con el hacer y con el futuro. A su vez, la "pareja presidencial" adquirió una importancia significativa, con la presencia de la mujer del presidente en múltiples actos protocolares - en los que no solo las revistas especializadas sino también los diarios y programas de diverso tipo comentan ampliamente su vestimenta- pero también en varias actividades vinculadas con la acción social, como visitas a comedores, guarderías, etcétera, junto a la ministra de Desarrollo Social, Carolina Stanley. Se realizó también una narración intensa de la vida familiar del presidente, en la que su hija más pequeña encontraría un lugar relevante. Como en otras latitudes, la vida privada del presidente despertó cada vez más interés, y su presentación pública se ofreció tanto en sus propias redes sociales como en las revistas locales e internacionales. ${ }^{17}$

Junto a la informalidad, la cercanía, el optimismo y la visibilización de la vida familiar, la acción comunicacional presidencial también se orientó a realzar la voluntad de escucha y de diálogo del gobierno con diferentes actores, en contraste con un activo jacobinismo de su predecesora. En este sentido, el presidente se presentaría a sí mismo como alguien que buscaba "cerrar la grieta" que desunía a los argentinos. La distancia con el estilo presidencial previo también se escenificó en la capacidad del presidente de cometer "errores" y pedir disculpas. Ante distintas medidas que generaron polémicas o movilizaciones (desde el nombramiento de jueces de la Corte Suprema por decreto hasta el aumento drástico de las tarifas de los servicios públicos), el presidente dio marcha atrás reconociendo lo errado de su decisión. La estrategia fue exitosa en distintas ocasiones, en tanto le permitió mostrarse como falible y humilde, al tiempo que constituyó un modo de avanzar por ensayo y error. En líneas generales, la imagen presidencial se mantuvo elevada a lo largo de todo el año, aunque sufrió una baja hacia fines de 2016 tanto en virtud de los problemas sociales y económicos del país como de la acumulación de esos errores que iban en contra de la imagen de cercanía y cuidado de la población. Según la consultora Poliarquía, la imagen positiva de Macri pasó de 64\% en diciembre de 2015 a 46\% en diciembre de 2016 (Poliarquía 2016). 
Gráfico 1. Imagen del presidente Macri, 2016

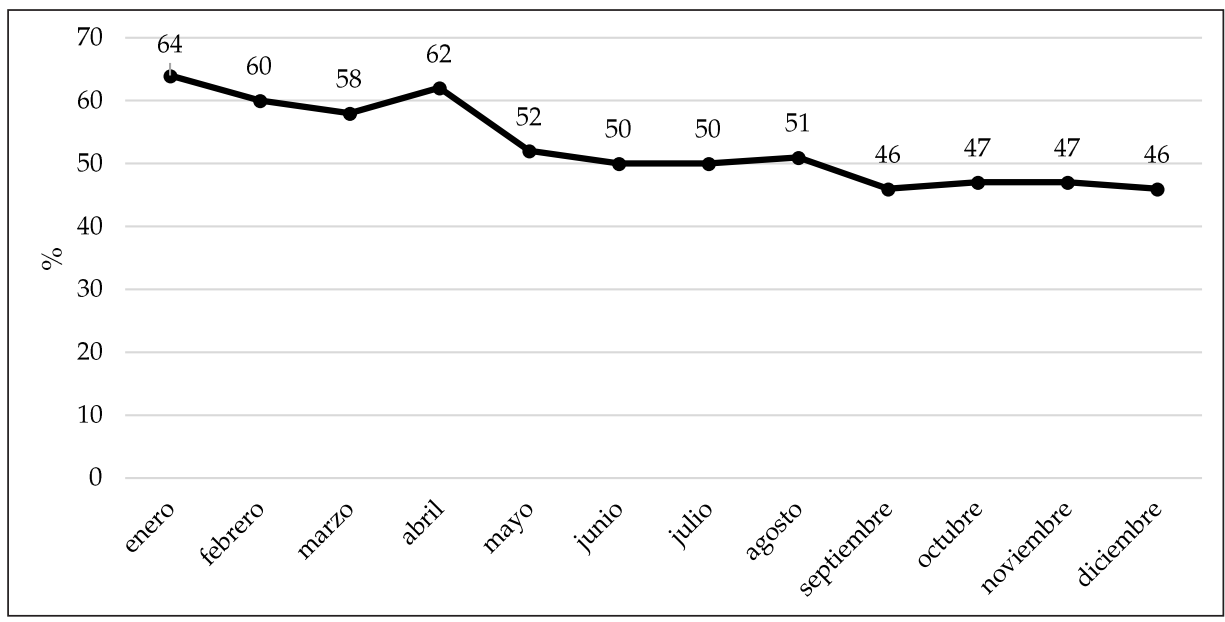

Fuente: Encuesta Nacional, Poliarquía (2016).

\section{ALIANZAS Y COALICIONES: EL CONGRESO Y EL MAPA FEDERAL}

El triunfo de Cambiemos en las presidenciales de 2015 trajo dos novedades fundamentales al escenario político-institucional argentino. Por un lado, el nuevo gobierno no contaría con mayoría en ninguna de las dos cámaras del Congreso. Por otro lado, el partido predominante de la coalición no poseía presencia territorial significativa en los veinticuatro distritos del país. De modo que tanto en la arena parlamentaria como en el gobierno del federalismo, debía establecer negociaciones y acuerdos con otras fuerzas políticas. Comencemos por el Congreso. Las divisiones en el interior del bloque del FPV, que comenzaron a producirse a partir de los primeros meses del año, permitieron al interbloque de Cambiemos convertirse en la primera minoría en la Cámara de Diputados (91/257 bancas). El kirchnerismo perdió diputados por decisión del peronismo de algunas provincias, como Misiones, de "despegarse" del FPV; en virtud del intento de otros dirigentes de formar una nueva línea interna partidaria, como la comandada por el exfuncionario Diego Bossio; y también por la salida de aquellos identificados con movimientos sociales, como el Movimiento Evita, que tomó distancia del liderazgo de Cristina Kirchner y estableció una estrategia política propia (ver Cuadro 3). Su debilitamiento impactó también en el funcionamiento del bloque en la Cámara de Senadores, donde a pesar de que el FPV mantuvo la mayoría - Cambiemos tiene apenas 15 de las 72 bancasprimó la posición conciliadora y dialoguista de los representantes de la mayor parte de las provincias gobernadas por el peronismo. 
Cuadro 3. Bloques Cámara de Diputados de la Nación, 2015-2016

\begin{tabular}{lccc}
\hline & \multicolumn{3}{c}{ Cantidad de bancas } \\
\cline { 2 - 4 } \multicolumn{1}{c}{ Partido } & $\begin{array}{c}2015 \\
\text { pre elecciones }\end{array}$ & $\begin{array}{c}2015 \\
\text { elecciones } \\
10 / 12 / 2015\end{array}$ & $\begin{array}{c}2016 \\
\text { redefinición bloques }\end{array}$ \\
\hline FPV y aliados & 131 & 95 & 81 \\
Cambiemos & 64 & 91 & 92 \\
UNA/Frente Renovador & 20 & 30 & 35 \\
PJ no K & 6 & 7 & 17 \\
PS/GEN/Progresistas & 13 & 9 & 6 \\
Izquierda & 3 & 4 & 4 \\
Otros & 20 & 21 & 22 \\
\hline
\end{tabular}

Fuente: elaboración propia en base a archivos de prensa y web de la HCDN.

Esa situación abrió la puerta para que Cambiemos encontrara diversos interlocutores con quienes negociar sus proyectos de ley. Para ello, actuó de manera cohesionada, con un funcionamiento que, en este caso, fue de coalición. A partir de la seguridad de los votos propios, entabló negociaciones con diferentes interlocutores: el peronismo renovador de Sergio Massa, el bloque del peronismo no kirchnerista de Bossio, algunos gobiernos provinciales que tienen bloques propios en las cámaras, como Santiago del Estero. Utilizó para ello dos caminos: la concesión de modificaciones a los proyectos de ley y el otorgamiento de recursos especiales a las provincias en las que el gobernador lograba sumar votos por fuera del FPV. Como sostiene Javier Zelaznik (2016), "el resultado fue un avance moderado y consensual de la agenda legislativa de gobierno" que le permitió avanzar con temas clave, como el pago a los holdouts y algunas incipientes reformas económicas. No está claro si este comportamiento colaborativo de algunos bloques no oficialistas se mantendrá en 2017, año electoral "en el que la diferenciación propia de la dinámica de la competencia choque con la cooperación propia de la dinámica del consenso" (Zelaznik 2016). Tampoco si será posible para Cambiemos aprobar leyes clave que hacen a su programa político, como aquellas vinculadas con el empleo y los impuestos. En todo caso, el mapa legislativo volverá a modificarse luego de las elecciones de medio término que tendrán lugar en octubre de 2017, y en buena parte el resultado de ellas dará una medida de las posibilidades del gobierno de avanzar con su proyecto.

En relación con el vínculo con las provincias, las atribuciones del Poder Ejecutivo para distribuir recursos a nivel subnacional que provenían del ciclo político anterior se mantuvieron, lo que le permitió asistir financieramente a las provincias gobernadas por Cambiemos, o aliadas con el oficialismo. ${ }^{18}$ Esta ayuda 
fue particularmente importante en el caso de la provincia de Buenos Aires, el distrito más grande del país, donde gobierna la dirigente de PRO María Eugenia Vidal. Allí, Cambiemos se juega buena parte de su suerte político-electoral en estos años. También las provincias de Jujuy y de Mendoza — gobernadas por Cambiemos - recibieron sumas elevadas, así como aliados recientes (Chubut, Santiago del Estero, Neuquén). Al mismo tiempo, el gobierno entregó recursos financieros e inició obra pública en otras provincias en virtud de negociaciones y compensaciones parlamentarias. En términos generales, el uso de esta atribución discrecional funciona en la democracia argentina reciente como un instrumento para conseguir gobernabilidad y apoyos legislativos en el contexto de su complejo federalismo.

En este contexto, cabe preguntarse si es posible robustecer la fuerza política propia a nivel nacional. Por un lado, las negociaciones con gobernadores de origen peronista pero circunstanciales aliados implicaron que el gobierno cediera el control de algunos puestos clave a nivel provincial vinculados con la gestión de dependencias y programas de política pública nacionales, lo que a corto plazo al menos mantiene recursos políticos en manos de las fuerzas políticas gobernantes en cada distrito, sea cual sea su signo partidario. ${ }^{19}$ Por otro lado, la tensión entre un partido dominante en el interior de la coalición Cambiemos con escasa presencia territorial más allá del centro del país, y el integrante más tradicional de ella, la Unión Cívica Radical, minoritario en la toma de decisiones a nivel central pero de gran presencia territorial, actualiza la paradoja del gobierno de Macri: para afianzarse como gobierno necesita de sus aliados, y al conservar los recursos de esos aliados, no puede crecer como fuerza política. Parece lejana la perspectiva de que, con los recursos que da el gobierno, PRO pueda consolidarse como partido a nivel nacional, como hizo a partir de 2007 en la ciudad de Buenos Aires. Eso lo vuelve dependiente de los radicales, a quienes no obstante mantiene relegados en la toma de decisiones, pero también abre la puerta para la incorporación de aliados de otras fuerzas, en especial peronistas, que pueden incorporarse a Cambiemos escapando de la crisis de su partido. Después de todo, PRO también está hecho de retazos de los partidos tradicionales. El problema es saber si las condiciones que le permitieron crecer como partido a nivel local están presentes, en la actualidad, para construir un partido nacional (Morresi y Vommaro 2014). Una respuesta positiva implicaría la constitución de una nueva fuerza con arraigo en todo el país capaz de disputar de igual a igual con los partidos tradicionales. Una respuesta negativa dejaría a PRO como una fuerza subnacional con dirigentes de proyección nacional, y mantendría su dependencia de esos partidos tradicionales, o al menos de algunas de sus facciones, para ser competitivo a nivel nacional.

19 Diferentes ministerios (Trabajo, Desarrollo Social) y dependencias oficiales (ANSES, PAMI) cuentan con oficinas en todas las provincias. Tradicionalmente, la fuerza política gobernante designaba en esos lugares a referentes propios en cada distrito. En este caso, el gobierno de Cambiemos no solo debió distribuir posiciones entre las fuerzas políticas que forman parte de la coalición, sino que también aceptó mantener cierto statu quo en provincias gobernadas por otros partidos. 


\section{PARA CONCLUIR: EL AÑO DE CAMBIEMOS}

El primer año de gobierno de Cambiemos reveló algunos rasgos centrales del nuevo ciclo político que se abrió en Argentina con la derrota el FPV en las presidenciales de 2015. La tensión entre el programa que la nueva coalición política viene a llevar a cabo y la necesidad de mantener la gobernabilidad política y social se volvió más acuciante por el carácter minoritario del nuevo gobierno; Cambiemos dio pruebas de una gran capacidad de negociación y de incorporación de aliados, aunque sea circunstanciales. Esta tensión y esta habilidad negociadora también parecen posponer las transformaciones que quiere llevar a cabo, en especial haciendo más gradual la baja del déficit fiscal y avanzando de manera más lenta en la presentación de iniciativas de reforma de tipo estructural. Eso hizo que 2016 haya sido, paradójicamente, un año de algunos triunfos políticos y bastantes fracasos económico-sociales. Hasta el último trimestre, la relación entre expectativas y problemas presentes daba un saldo más que favorable para el gobierno; la tendencia comenzó a revertirse desde entonces, y no está claro el camino por el que podrá volver a pedir sacrificios a la población a cambio de un futuro promisorio. Por ahora, la estrategia seguida fue la de polarizar con el kirchnerismo, desprestigiado en buena parte de la ciudadanía, como modo de construirse como garantía de una no vuelta al pasado. Los escándalos de corrupción denunciados a lo largo de 2016 terminaron por asociar el ciclo político anterior para buena parte de la opinión pública con un período de despilfarro y uso de los fondos públicos para fines partidarios.

No obstante, la defensa de diferentes actores de ciertos derechos sociales consolidados en el ciclo político precedente da cuenta de que la relación con el pasado no es únicamente de condena. La necesidad del nuevo gobierno de producir escasas innovaciones en materia de políticas sociales (Niedzwiecki y Pribble 2017) y el fomento de diálogo con los actores sindicales, en un año de aumentos salariales levemente inferiores al período anterior, muestra que las realidades heredades van más allá del movimiento que gobernó al país entre 2003 y 2015. Al mismo tiempo, el nuevo gobierno se topa con algunos problemas económicos estructurales — como la baja tasa de inversión privada de mediano y largo plazo, la dificultad para generar una industria competitiva y los cuellos de botella por escasez de divisas- que trascienden los estilos de gobierno. Si la negociación legislativa y a nivel de gobiernos subnacionales con un sector del peronismo no kirchnerista permitió destrabar algunas leyes fundamentales, la relación con los actores productivos - a excepción de los productores agropecuarios y los bancos, grandes beneficiarios de los cambios económicos recientes- parece estancada.

Entretanto, un cambio en la comunicación política trajo un estilo menos confrontativo que prometía terminar con la polarización. Sin embargo, es en la polarización con el kirchnerismo que el nuevo gobierno obtiene sus mayores réditos en términos de comunicación política y de apoyos ciudadanos. Es 
probable que tenga incentivos para mantener entonces esta "grieta", aunque lo haga con un estilo más informal y de cercanía. Las dificultades económicas y las necesidades políticas, por otra parte, parecen postergar el final de la democracia delegativa. Aunque Cambiemos asumió con una promesa republicana de límite del Poder Ejecutivo, en buena parte, la lógica de funcionamiento del poder en Argentina lleva a los poderes ejecutivos a construirse fuertes. Necesitan acumular poder. $Y$, en tren de acumular, un proyecto político comienza a creerse duradero. No parece que Cambiemos quiera ser una excepción. Su proyecto de país requiere de ese tiempo medianamente largo de la historia. Así se entiende que, terminada la campaña que fue también una larga marcha y, a poco de asumir, el nuevo presidente ya haya usado su poder para reorganizar ministerios y redefinir atribuciones por decreto, aun contra leyes sancionadas por el Congreso, como en el mencionado caso de la Ley de Medios. En definitiva, en materia institucional, la voluntad de cambio pretende deshacer la herencia kirchnerista: aquí, el republicanismo comenzaría luego de la ruptura con la normalidad pasada, un Ancien Régime a desterrar.

\section{REFERENCIAS}

Aboy Carlés, Gerardo. 2001. Las dos fronteras de la democracia argentina. Rosario: Homo Sapiens. Becerra, Martín. 2016. "Restauración: cambios en las políticas de comunicación." Épocas. Recuperado el 6 de marzo de 2017 de: http://revistaepocas.com.ar/restauracion-cambios-en-las-politicas-de-comunicacion/

Bonvecchi, Alejandro y Agustina Giraudy. 2007. “Argentina: crecimiento económico y concentración del poder institucional." Revista de Ciencia Política 27: 29-42.

CIDH. 2016, 2 de diciembre. "CIDH urge al Estado argentino a responder el caso de Milagro Sala." Comunicado de prensa. Recuperado el 10 de agosto de 2017 de: http://www. oas.org/es/cidh/prensa/comunicados/2016/182.asp

Cruz, Facundo y Lara Goyburu. 2016, 2 de octubre. "Cambiemos, esa coalición de nuevo tipo." La Nación. Recuperado el 1 de agosto de 2017 de: http://www.lanacion.com. ar/1942551-cambiemos-esa-coalicion-de-nuevo-tipo

Diamand, Marcelo. 1973. Doctrinas económicas, desarrollo e independencia. Buenos Aires: Paidós.

Diario Popular. 2015, 17 de marzo. "Macri no quiere un gobierno de coalición con la UCR." Diario Popular. Recuperado el 1 de agosto de 2017 de: https://www.diariopopular. com.ar/politica/macri-no-quiere-un-gobierno-coalicion-la-ucr-n219738

Etchemendy Sebastián. 2001. "Construir coaliciones reformistas: la política de las compensaciones en el camino argentino hacia la liberalización económica." Desarrollo Económico 40(160): 675-706.

Fernández Blanco, Pablo. 2017, 11 de marzo. “El agua aumentará 23\% y confirman topes de hasta 500\% para la suba del gas." La Nación. Recuperado el 1 de agosto de: http:// www.lanacion.com.ar/1992123-el-agua-aumentara-23-y-confirman-topes-de-hasta500-para-la-suba-del-gas

Hauser, Irina. 2016, “Justicia Federal: Comodoro en llamas." Anfibia. Recuperado el 1 de agosto de 2017 de: http:/ / www.revistaanfibia.com/cronica/comodoro-en-llamas/

Iglesias Illia, Hernán. 2015. Cambiamos. Buenos Aires: Editorial Sudamericana.

INDEC. 2017. Estadísticas de registros laborales. Puestos de trabajo y remuneraciones de los asalariados registrados Tercer trimestre de 2016. Informes Técnicos 1(5). Cuentas nacionales 1(1). Buenos Aires: Ministerio de Hacienda.

Leiras, Marcelo e Inés Cruzalegui. 2009. “Argentina: problemas macroeconómicos, conflicto social y debilitamiento de la coalición de gobierno." Revista de Ciencia Política 29(2): 223-246. 
Morresi, Sergio y Gabriel Vommaro. 2014. "Argentina: The difficulties of the Partisan Right and the Case of Propuesta Republicana." En The Resilience of the Latin American Right, editado por Juan Pablo Luna y Cristóbal Rovira Kaltwasser. Baltimore: The Johns Hopkins University Press, 319-342.

Murillo, María Victoria. 1997. "La adaptación del sindicalismo argentino a las reformas de mercado en la primera presidencia de Menem." Desarrollo Económico 37(147): 419-446.

Murillo, María Victoria, Julia María Rubio y Jorge Mangonnet. 2016. “Argentina: el protagonismo de los votantes y la alternancia electoral." Revista de Ciencia Política 36(1): 3-26.

Niedzwiecki, Nadia y Jennifer Pribble. 2017. "Social Policies and Center-Right Governments in Argentina and Chile." Latin American Politics and Society 59(3): 72-97.

Obarrio, Mariano. 2016, 21 de julio. "Otorgan más recursos a las provincias del oficialismo. Los cinco distritos recibieron $41 \%$ de los fondos que distribuyó hasta ahora el Ministerio del Interior." La Nación. Recuperado el 1 de agosto de 2017 de http:/ /www. lanacion.com.ar/1920372-otorgan-mas-recursos-a-las-provincias-del-oficialismo

Pereyra, Sebastián. 2013. Política y transparencia. Buenos Aires: Siglo XXI.

Poliarquía. 2016. "Encuesta nacional. Informe mensual de situación sociopolítica de la Argentina." Buenos Aires, diciembre.

Rosso, Fernando. 2016, 8 de diciembre. "Andrés Malamud: 'El peronismo es el elefante en el bazar de la política argentina.'” La Izquierda. Recuperado el 1 de agosto de 2017 de: http:/ / www.laizquierdadiario.com/Andres-Malamud-El-peronismo-es-el-elefante-en-el-bazar-de-la-politica-argentina

Smulovitz, Catalina. 2008. "La política por otros medios. Judicialización y movilización legal en la Argentina." Desarrollo Económico 48(190/191): 287-305.

Vommaro, Gabriel. 2017. "Política popular en tiempos de economías postindustriales: trabajo territorial y economía moral en la Argentina reciente." Revista Pós Ciências Sociais 14(27): 77-98.

Vommaro, Gabriel y Melchor Armesto. 2015. “¿Nuevos políticos en el partido, viejos políticos en las listas? Reclutamiento partidario y división del trabajo político en PRO, en la Ciudad Autónoma de Buenos Aires." Pasado Abierto. Revista del CEHis 2: 110-132.

Vommaro, Gabriel, Sergio Morresi y Alejandro Bellotti. 2015. Mundo PRO. Buenos Aires: Planeta.

Zelaznik, Javier. 2016, 19 de diciembre. "Macri en el Congreso." Bastión digital. Recuperado el 6 de marzo de 2017 de http:/ / ar.bastiondigital.com/notas/macri-en-el-congreso

Gabriel Vommaro es Investigador del CONICET e Investigador-docente en la Universidad Nacional de General Sarmiento (Instituto del Desarrollo Humano, Área de Política). Sus temas de investigación son: cultura, prácticas y organizaciones políticas en la Argentina reciente; medios de comunicación y política; ciencias sociales y expertise. Sus artículos y ensayos de investigación fueron publicados en diversas revistas de ciencias sociales, entre ellas: Actes de la Recherche en Sciences Sociales, Journal of Latin American Studies, Politix, Cahiers des Amériques Latines, Apuntes de Investigación del CECYP, Revista Pós Ciências Sociais y Desacatos. Revista de Antropología Social. Es además autor de varios libros. Email: gvommaro@ungs.edu.ar.

Mariana Gené es Doctora en Ciencias Sociales por la UBA y en Sociología Política por la Ecole des Hautes Etudes en Sciences Sociales de Paris. Es investigadora asistente del CONICET con sede en el Instituto de Altos Estudios Sociales de la Universidad Nacional de San Martín (IDAES-UNSAM) y docente de la Universidad de Buenos Aires, la Universidad Nacional de General Sarmiento y la Universidad Nacional de San Martín. Sus temas de investigación son: profesión política, agencias estatales y socio-historia de la Argentina reciente. Compiló junto a Gabriel Vommaro el libro La vida social del mundo político (2017) y ha publicado numerosos artículos en revistas nacionales y extranjeras. Email: mariana.gene@yahoo.com.ar. 
- 